\title{
Robert Mallet, formaliste de la confidence
}

\author{
Jacques Arnold
}

$\mathrm{R}$ obert Mallet associe depuis toujours dans sa manière d'être ouverture d'esprit Cn partie une vertu mulltaire car, si son oeuvie est discrete à ce propos, in importe en partie une vertu militaire car, si son oeuvre est discrète à ce propos, il importe avant toute chose de rappeler que Robert Mallet fit la guerre et qu'il s'est intégré à la Résistance française. Avoir été homme de radio, administrateur universitaire. être présent dans la vie littéraire actuelle comme romancier, critique, voilà ce qu'on pourrait appeler l'infrastructure de Robert Mallet poète, car avant toutes les susdites activitès et tous les autres effets de sa créativité, Robert Mallet s'est affirmé comme poète de son entrée dans la carrière jusqu'à ce jour. Il est signataire d'une bonne vingtaine de recueils de poésie.

Robert Mallet est de la catégorie des poètes qui ne veulent pas être simplement le médium mystérieux d'un discours prétendant à la fois défier l'explication et s'en défier. A cet égard il n'est pas une exception parmi les poètes français contemporains quelle que soit la bande d'âge. Il n'ignore pas toutefois la tyrannie des structures et, tout en s'en gardant, se laisse séduire - s'est laissé séduire quelques fois par le charme des concaténations spontanées:

prends-la telle qu'elle est

sinon tu te fals prendre

comme un morceau de viande

au sexe d'un collet (Quand le miroir s'étonne).

Cela prouve qu'il côtole bien sans cesse le mystère s'il s'ingénire à n'en être pas dupe. C'est une attitude poétique de base.

Depuis Mahafaliennes (Gallimard, 1961), Robert Mallet s'est complu à fournir au lecteur les clés de ses poèmes. Il ne leur donne pas de titre comme si le titre était un instrument d'effraction, il expose en quelques mots un argument dont la nature est assez variable, la source en tout cas fortuite et qui, le plus souvent est situé, c'est-à-dire accompagné d'un nom de lieu où l'ẻvẻnement, la circonstance, l'observation, la sensation, l'émotion se sont produits. Le poète livre ainsi au lecteur une confidence qui est donc marque de confiance en lui pour la lecture à venir du poème.

La présentation typographique des poèmes de Robert Mallet demeure tout à fait traditionnelle jusque vers les années 80 alors que la facture proprement dite a évolué depuis ses débuts dans le sens d'une libération de certaines formes, à savoir la métrique syllabique et la rime. Le souci de la forme - qui pour certains se manifeste comme souci de l'informe - est une caractéristique de la "modernité" des temps actuels. Le fait de mépriser ou de maltraiter ou d'ignorer "superbement" les modes prosodiques et les rythmes traditionnels (ceux de lalexandrin, du décasyllabe, de l'octosyllabe classiques, par exemple), l'anarchie du dispositif 
formel, trop souvent suspect d'inconscience malheureusement, sont des signes flagrants d'intérêt pour les formes. Paradoxe apparent? mais les iconoclastes sont souvent des passionnés de ce qu'ils brisent. Ceci devrait suffire à expliquer l'attention qu'll convient d'accorder au problème des formes dans la poésie de Robert Mallet. Mais le poète n'a pas manqué de s'exprimer lui-même sur ce sujet, entre autres dans Apostilles (Gallimard, 1972), recueil de pensées, réflexions, boutades, maximes très révélatrices du personnage et donc précieuses pour décrypter sa poésie. En ce qui concerne la forme, Robert Mallet se montre dans cet ouvrage à la fois subtil et péremptoire:

Par le mépris des formes, c'est le fond qu'ils lèsent. S'ils sont contre les formes au nom de la simplicité, rien ne devrait les empêcher d'inventer des formes simples (p. 71).

L'idée maîtresse de Robert Mallet apparait ici clairement. Il ne s'agit pas de jeter l'anathème sur ceux qui sont en quête d'invention et la preuve en est dans cette autre réflexion:

On a disloqué le vieux moule. On l'a moqué, banni. Née de ce refus et de ce mépris, la poésie s'est enrichie. Les poèmes n'ont pas été plus beaux (p. 203).

Auparavant Robert Mallet avait fait une "discrète" allusion à la querelle des tenants du vers classique et des verslibristes en général qui fait évidemment partie des vieilles lunes mais ne manque pas de resurgir à l'occasion dans nos provinces et les plus "prestigieux" quartiers de Paris:

La beauté ne salt pas mieux se passer de règles que de liberté. Le vers le plus strict est toujours libre, pourvu qu'harmonleux (p. 203).

Cette sentence est précédée d'une constatation quil révèle bien toute la pensée du moraliste critique sur cette question de la forme:

Qu'avec le plus arbitrairement factice - la rime, les pieds

- on puisse faire le plus émouvant, c'est là le miracle (p. 203).

Inversées dans leur ordre pour les besoins de la cause, les citations ci-dessus dẻnotent le même souci: l'écriture poétique ne peut aller sans discipline. Une dernière réflexion doit encore éclairer plus nettement la position de Robert Mallet:

L'inspiration du poète est la plus asservie. Si elle subit trop les règles des autres, cesse le mystère. Si elle en fait fi, 
cesse la communication. La combinaison neuve existe alors, mals sans objet. sinon l'évasion du poète (p. 202).

Trouver sa voie entre trop de clartê formelle et trop de singularité intime apparait comme l'une des ambitions majeures du poète Robert Mallet. Il y a satisfait par la pratique des deux procédés: l'argument *orienteur du poème et la disposition typographique.

Le pôle directeur du message que Robert Mallet entend faire passer se nomme franchise. Il ne faut pas négliger le fait qu'il a intitulé son premier receuil Sincérités (Revue septentrionale, 1936). Dix ans plus tard dans L'égoïste clé (Robert Laffont. 1946) se fait jour un propos analogue plein de fougue encore et d'enjouement juvénile qui résume prémonitoirement ce qu'est devenue la démarche poétique du personnage protéiforme qui aurait pu porter tant de masques.

Robert Mallet dès cette époque prend bien clairement le parti du lyrisme personnel en quoi 11 rejoint une nombreuse cohorte, sans doute la plus nombreuse de toutes celles qui rassemblent les pratiquants de la poésie. On ne peut s'y distinguer que par la manière et c'est sur ce lieu commun que, faisant la nique dirait-on au surréalisme comme aux fantaisistes, comme à Prévert, comme à Fombeure en ces années de l'immédiat après-guerre (1946), le jeune poète conclut son Egoïste clé dans le dernier poème, en écho au premier:

Au moule des poèmes vibre mon plus secret qui se veut libre. et je me purge de mes maux par la grâce des jeux de mots (Jeux de mots).

Ce ton désinvolte se pérennise dans Semer l'arbre (Gallimard, 1991). Ce qui demeure aussi dans ce dernier ouvrage, c'est un goût de la sentence:

L'amour ne se veut pas
c'est luil quil nous choisit
il vit de nous vouloir
sans que nous le voulions
survit sans le savoir de savoir s'inventer
et meurt par trop de volonté
(p. 63).

La véritê du poète combine alors le rythme avec un humour sous-jacent d'où émerge maintes fois une mélancolie pudique:

Le bonheur

se reçolt et se donne

il ne s'use pas nous l'usons (p. 33). 
A travers le subtil agencement des découpes et des sonoritês dans ces poèmes, on ne peut manquer de s'interroger sur leur finalité. Ne s'agirait-il pas au fond d'une recherche de l'unité dans la discontinuité? Ne serait-ce que par les confidences des arguments orienteurs qui parsèment ses recueils il apparait clairement que le personnage en tant qu'individu s'est souvent senti éclatê: ce qui le fait tenir ensemble, à son sentiment profond, c'est d'avoir été poète et de l'être resté. En avançant dans la vie, certains domaines de la thématique du lyrisme universel, lamour-passion par exemple, se sont estompés; il semble que les questions plus "métaphysiques" prennent le pas sur les autres, encore que...Les signes de l'addition (Gallimard, 1953) s'y adonnent déjà sans retenue. Il conviendra cependant de monter en épingle que le formalisme rythmique et typographique façonné par le poète s'accommode fort bien d'un lyrisme de pensée, de méditation morale, de jugement où se marient sentiment et raison, de sentences où transparaissent selon les cas la colère, l'amertume, la dérision et le mépris, sans néglier l'humour qui peut aller avec. En nombre de ses poèmes Robert Mallet défie l'inconnaissable: comment le monde peut-1l être sans l'homme, comme l'homme peut-il être sans le monde? C'est pourquol dans un article récent de La Sape $\left(\mathrm{n}^{\circ} 34 / 35\right.$, décembre 1993) à propos de Semer l'arbre, Serge Brindeau propose "une lecture philosophique" de cette oeuvre poétique effectivement foisonnante de questions sur le temps, l'éternité. les êtres et l'Etre, tout ce qui découle de là ou y aboutit.

Où situer Robert Mallet? Il ne faudrait pas se limiter pour une juste appréciation des choses à ce formalisme et ce penchant à la confidence, car la singularité de Robert Mallet constitue l'une des tendances de la vision poétique contemporaine qu'il parait impossible de négliger. A l'écart des attitudes dédaigneuses concernant le sens comme de la dérision travestie qui inspire le néo-classicisme ou encore de la désinvolture qui laisse au lecteur tout le travail à faire, Robert Mallet poète s'efforce de raison garder. Au volume II de LaPoésie française duXXè siècle, Robert Sabatier le loge dans une section intitulée "Eloge de la diversité." C'est dire que Robert Mallet ne se laisse inféoder à aucun des courants dont 11 a été et/ou est toujours le contemporain.

On en jugera sans mal à la lecture des deux poèmes inédits (voir p. 147) choisis et préfacés par Robert Mallet sans rien savoir de l'option adoptée dans le présent article. Il pose là les questions: qu'est-ce que l'amour? Qu'est-ce que la (ma) vie? En confidence il vous donne sa réponse dans les formes que vous savez. Il prend un risque qui n'est pas mineur. C'est aussi une forme de son courage congénital dont Robert Mallet a fait montre en toutes les circonstances de sa vie. 